\title{
Upward Geometric Graph Embeddings into Point Sets ${ }^{\star}$
}

\author{
Patrizio Angelini ${ }^{1}$, Fabrizio Frati ${ }^{1}$, Markus Geyer ${ }^{2}$, Michael Kaufmann ${ }^{2}$, \\ Tamara Mchedlidze ${ }^{3}$, and Antonios Symvonis ${ }^{3}$ \\ 1 Dipartimento di Informatica e Automazione - Università Roma Tre, Italy \\ \{angelini, frati\}adia.uniroma3. it \\ 2 Wilhelm-Schickard-Institut für Informatik - Universität Tübingen, Germany \\ \{geyer, mk\}@informatik. uni-tuebingen. de \\ ${ }^{3}$ Dept. of Mathematics, National Technical University of Athens, Athens, Greece \\ \{mchet, symvonis\}@math.ntua.gr
}

\begin{abstract}
We study the problem of characterizing the directed graphs with an upward straight-line embedding into every point set in general or in convex position. We solve two questions posed by Binucci et al. [Computational Geometry: Theory and Applications, 2010]. Namely, we prove that the classes of directed graphs with an upward straight-line embedding into every point set in convex position and with an upward straight-line embedding into every point set in general position do not coincide, and we prove that every directed caterpillar admits an upward straight-line embedding into every point set in convex position. Further, we provide new partial positive results on the problem of constructing upward straight-line embeddings of directed paths into point sets in general position.
\end{abstract}

\section{Introduction}

Constructing planar straight-line embeddings of graphs into point sets is a well-studied topic of research since more than twenty years. A celebrated result of Gritzmann et al. [9] is that the class of graphs that admit a planar straight-line embedding into every point set in general position or in convex position is the one of the outerplanar graphs. Efficient algorithms are known to embed outerplanar graphs [4] and trees [5] into any point set in general or in convex position. Further, while testing whether a graph admits a planar straight-line embedding into every point set in general or in convex position can be done efficiently, due to the above cited characterization [9] and to the existence of a linear-time algorithm to test whether a graph is outerplanar [11], testing whether a graph admits a planar straight-line embedding into a given point set in general position is $\mathcal{N} \mathcal{P}$-hard, as proven by Cabello [6]. Planar graph embeddings into point sets have been also studied when edges are allowed to bend (see, e.g., [10|2|7]).

The problem of constructing upward planar straight-line embeddings of directed graphs into point sets has been first suggested by Giordano et al. [8] and has been very recently tackled by Binucci et al. in [3], who proved the following main results: (a) No biconnected directed graph admits an upward planar straight-line embedding into

\footnotetext{
* This work was partially supported by MIUR (Italy), Projects AlgoDEEP number 2008TFBWL4 and FIRB "Advanced tracking system in intermodal freight transportation" number RBIP06BZW8, by the German Research Foundation (DFG), project KA 812/15-1 'Graph Drawing for Business Processes', and by the National Technical University of Athens research program $\Pi E B E 2008$.
}

U. Brandes and S. Cornelsen (Eds.): GD 2010, LNCS 6502, pp. 25-37 2011.

(C) Springer-Verlag Berlin Heidelberg 2011 
every point set in convex position; (ii) the upward planar straight-line embeddability of a directed graph into every one-side convex point set can be characterized and efficiently tested; (iii) there exist directed trees that do not have an upward planar straight-line embedding into every point set in convex position; (iv) every directed path admits an upward planar straight-line embedding into every point set in convex position.

In this paper we continue the study of the straight-line embeddability of directed graphs into planar point sets and show the following results.

In Sect. 3, we study upward planar straight-line embeddings of directed graphs into point sets in general and in convex position. First, we solve an open problem posed in [3], by exhibiting an infinite class of upward planar directed graphs admitting an upward planar straight-line embedding into every point set in convex position, but not into every point set in general position, showing an interesting difference between upward planar straight-line embeddability of directed graphs and planar straight-line embeddability of undirected graphs, as the classes of graphs with a planar straight-line embedding into every point set in convex position and into every point set in general position coincide. Second, we show that every single-source upward planar directed graph with cycles of length at most three admits an upward planar straight-line embedding into every point set in general position. Such a result is the best possible with respect to the number of sources and to the length of the longest cycle.

In Sect. 4 we study upward planar straight-line embeddings of directed trees into point sets in convex position. We solve an open problem posed in [3] by proving that every directed caterpillar admits an upward planar straight-line embedding into every point set in convex position, improving the result in [3] stating that every directed path admits an upward planar straight-line embedding into every point set in convex position.

In Sect. 5, we study upward planar straight-line embeddings of directed paths into point sets in general position. We tackle the problem by considering directed paths with few switches (a switch is either a source or a sink). While the upward planar straightline embeddability of directed paths with at most two or three switches into point sets in general position can be trivially proven, it is already difficult to deal with directed paths with four or five switches. We prove that directed paths with four (or five) switches admit an upward planar straight-line embedding into every point set in general position, if we suppose that at least one (at least two) of the monotone paths composing the directed paths with four (or five) switches are single edges. Finally, we show that every directed path with at most $k$ switches admits an upward planar straight-line embedding into every point set in general position with $n 2^{k-2}$ points.

Omitted proofs can be found in the full version of the paper [1].

\section{Preliminaries}

A point set in general position, or general point set, is such that no three points lie on the same line and no two points have the same $y$-coordinate. The convex hull $C h(S)$ of a point set $S$ is the point set that can be obtained as a convex combination of the points of $S$. A point set in convex position, or convex point set, is such that no point is in the convex hull of the others. In a point set $S$, each point $p \in S$ is given by its coordinates $x(v)$ and $y(v)$ in the plane. We denote by $b(S)$ and by $t(S)$ the lowest and the highest point of $S$, respectively. A one-side convex point set $S$ is a convex point set in which 
$b(S)$ and $t(S)$ are adjacent in the border of $C h(S)$. During the execution of an algorithm which embeds a graph $G$ into a point set $S$, a free point is a point of $S$ to which no vertex of $G$ has been mapped yet. Given a point $p$ in a point set $S$, a subset $S^{\prime}$ of $S$ is clockwise separated around $p$ if a half-line fixed at $p$, starting from a horizontal position, directed towards decreasing $x$-coordinates, and moving clockwise encounters all the points of $S^{\prime}$ before encountering any other point of $S$. A counterclockwise separation is defined symmetrically.

An upward planar directed graph admits a planar drawing where each edge is represented by a curve monotonically increasing in the $y$-direction. In the following we refer to paths, cycles, caterpillars, and trees meaning upward planar directed graphs whose underlying graphs are paths, cycles, caterpillars, and trees, respectively.

An upward straight-line embedding of a graph into a point set is a mapping of each vertex to a distinct point and of each edge to a straight-line segment between its endpoints such that no two edges cross and each edge $(u, v)$ has $y(u)<y(v)$.

A monotone path $\left(v_{1}, v_{2}, \ldots, v_{k}\right)$ is such that edge $\left(v_{i}, v_{i+1}\right)$ is directed from $v_{i}$ to $v_{i+1}$, for $1 \leq i \leq k-1$. An upward straight-line embedding of a monotone path into any general point set $S$ can be easily constructed by mapping vertex $v_{i}$ to the $i$-th lowest point of $S$. A monotone path is trivial when it consists of a single edge.

\section{Embeddings of Directed Graphs into Point Sets}

In this section we study the relationship between upward straight-line graph embeddability into convex point sets and upward straight-line graph embeddability into general point sets, and the relationship between upward straight-line graph embeddability, the number of switches, and the length of the longest cycle in the underlying graph.

First, we show an infinite class of graphs that admit an upward straight-line embedding into every convex point set but not into every general point set.

Let $G_{k}$ be defined as follows, for every $k \geq 3: G_{k}$ has $3 k$ vertices, it contains a 3 -cycle $C^{3}$ composed of edges $(u, v),(v, z)$, and $(u, z)$, and it contains 4 -cycles $C_{i}^{4}$, with $i=1, \ldots, k-1$, composed of edges $\left(u, v_{i}\right),\left(v_{i}, w_{i}\right),\left(w_{i}, z_{i}\right)$, and $\left(u, z_{i}\right)$.

Lemma 1. $G_{k}$ admits an upward straight-line embedding into every convex point set $S$ with $3 k$ points.

Proof: Map $u$ to $b(S)$. Let $l$ and $r$ be the number of points in the subsets $L$ and $R$ of $S$ to the left and to the right, respectively, of the line through $b(S)$ and $t(S)$.

If $l \equiv 0 \bmod 3($ and $r \equiv 1 \bmod 3)$, then iteratively map $u_{i}, v_{i}$, and $z_{i}$ to the lowest three free points of $L$, for $i=1,2, \ldots, \frac{l}{3}$; further, iteratively map $u_{i}, v_{i}$, and $z_{i}$ to the lowest three free points of $R$, for $i=\frac{l+3}{3}, \frac{l+6}{3}, \ldots, k-1$; finally, map $v$ and $z$ to the highest point of $R$ and to $t(S)$, respectively. If $l \equiv 1 \bmod 3($ and $r \equiv 0 \bmod 3)$, then iteratively map $u_{i}, v_{i}$, and $z_{i}$ to the lowest three free points of $L$, for $i=1,2, \ldots, \frac{l-1}{3}$; further, map $v$ and $z$ to the highest point of $L$ and to $t(S)$, respectively; finally, iteratively map $u_{i}, v_{i}$, and $z_{i}$ to the lowest three free points of $R$, for $i=\frac{l+2}{3}, \frac{l+5}{3}, \ldots, k-1$. If $l \equiv 2 \bmod 3($ and $r \equiv 2 \bmod 3)$, then iteratively map $u_{i}, v_{i}$, and $z_{i}$ to the lowest three free points of $L \cup\{t(S)\}$, for $i=1,2, \ldots, \frac{l+1}{3}$; further, iteratively map $u_{i}, v_{i}$, and $z_{i}$ to the lowest three free points of $R$, for $i=\frac{l+4}{3}, \frac{l+7}{3}, \ldots, k-1$; finally, map $v$ and $z$ to the highest two points of $R$. 


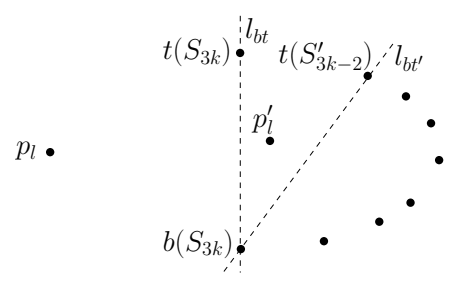

Fig. 1. Point set $S_{3 k}$

In all the cases, the obtained straight-line embedding is upward and planar.

Lemma 2. There exists a general point set $S_{3 k}$ with $3 k$ points such that $G_{k}$ does not admit any upward straight-line embedding into $S_{3 k}$.

Proof: Point set $S_{3 k}$ is any point set that satisfies the following constraints (see Fig. 1). One point $p_{l}$ is to the left of the line $l_{b t}$ through $b\left(S_{3 k}\right)$ and $t\left(S_{3 k}\right)$. The remaining $3 k-3$ points are to the right of $l_{b t}$ and, together with $b(S)$, they form a convex point set $S_{3 k-2}^{\prime}$ with one point $p_{l}^{\prime}$ lying to the left of the line $l_{b t^{\prime}}$ through $b(S)$ and $t\left(S_{3 k-2}^{\prime}\right)$.

Observation 1. Let $G$ be a graph containing a 4-cycle $C$ composed of edges $\left(x_{1}, x_{2}\right)$, $\left(x_{2}, x_{3}\right),\left(x_{3}, x_{4}\right)$, and $\left(x_{1}, x_{4}\right)$. Let $S$ be a point set such that exactly one point $p_{l}(S)$ lies to the left of the line through $b(S)$ and $t(S)$. Suppose that an edge of $G$ has been mapped to segment $\overline{b(S) t(S)}$. Then, there exists no upward embedding of $G$ into $S$ in which a vertex of $C$ is mapped to $p_{l}(S)$.

Since $u$ is the only source of $G_{k}$, such a vertex has to be mapped to $b\left(S_{3 k}\right)$. Further, since a sink of $G_{k}$ has to be mapped to $t\left(S_{3 k}\right)$ and since every sink of $G_{k}$ is adjacent to $u$, segment $\overline{b\left(S_{3 k}\right) t\left(S_{3 k}\right)}$ is part of any embedding. Then, by Observation 1 no vertex of a 4 -cycle $C_{i}^{4}$ of $G_{k}$ is mapped to $p_{l}$. Hence, a vertex of $C^{3}$ is mapped to $p_{l}$. If such a vertex is $z$, then vertex $v$ is mapped to a point to the right of $l_{b t}$, hence $\overline{b(S) t(S)}$ crosses the segment between $z$ and $v$. It follows that $v$ is mapped to $p_{l}$. If $z$ is not mapped to $t\left(S_{3 k}\right)$, then $\overline{b\left(S_{3 k}\right) t\left(S_{3 k}\right)}$ crosses the segment between $z$ and $v$. Hence, $z$ is mapped to $t\left(S_{3 k}\right)$. Then, all the vertices of the 4 -cycles of $G_{k}$ are mapped to the vertices of $S_{3 k-2}^{\prime}$. A sink of one of the 4-cycles has to be mapped to $t\left(S_{3 k}^{\prime}\right)$. Since every sink of $G_{k}$ is adjacent to $u$, segment $\overline{b\left(S_{3 k}\right) t\left(S_{3 k}^{\prime}\right)}$ is part of any embedding. Then, by Observation 1 no vertex of a 4 -cycle $C_{i}^{4}$ of $G_{k}$ can be mapped to $p_{l}^{\prime}$, thus proving the lemma.

We get the following:

Theorem 1. For every $k \geq 3$, there exists a $3 k$-vertex upward planar digraph that admits an upward straight-line embedding into every convex point set with $3 k$ points but not into every general point set with $3 k$ points.

Next, we show that every single-source graph $G$ whose every simple cycle has length three admits an upward straight-line embedding into every general point set. Such a result is tight both with respect to the maximum length of a cycle in $G$ and with respect to the number of sources in $G$. Namely, a single-source graph exists whose every simple 


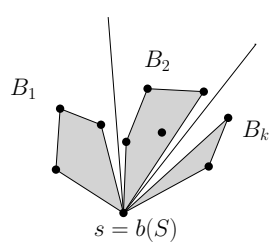

(a)

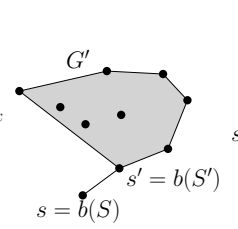

(b)

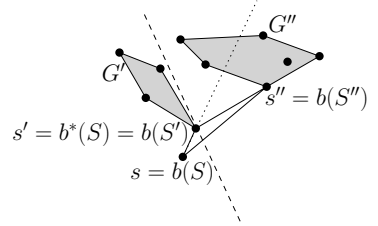

(c)

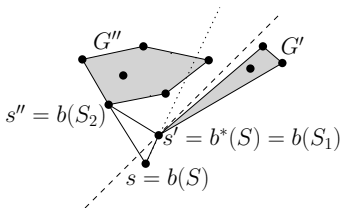

(d)

Fig. 2. (a) $s$ is a cut-vertex. (b) $B$ is an edge. (c) $B$ is a 3 -cycle $\left(s, s^{\prime}, s^{\prime \prime}\right)$ and $l$ encounters $b\left(S^{\prime \prime}\right)$ after $s$. (d) $B$ is a 3 -cycle $\left(s, s^{\prime}, s^{\prime \prime}\right)$ and $l$ encounters $b\left(S^{\prime \prime}\right)$ before $s$.

cycle has length at most four not admitting any upward straight-line embedding into some general point set (by Lemma 2). Further, a graph with two sources exists whose every simple cycle has length three not admitting any upward straight-line embedding into some general point set (by the results in [3] on upward straight-line embeddability into one-side convex point sets).

We show a recursive algorithm to construct upward straight-line embeddings of single-source graphs whose every simple cycle has length three into every general point set. The recursion is on the number $x$ of biconnected components of $G$. If $x=1$, then the statement is trivially true. If $x>1$, consider the unique source $s$ of $G$.

If $s$ is a cutvertex of $G$, denote by $B_{1}, \ldots, B_{k}$ the connected components obtained by removing $s$ from $G$ (see Fig. 2(a)). Clockwise separate the sets $S_{1}, \ldots, S_{k}$ with $\left|B_{1}\right|, \ldots,\left|B_{k}\right|$ points, respectively, around $b(S)$. Recursively construct straight-line embeddings of the subgraphs of $G$ induced by the vertices in $B_{1} \cup\{b(S)\}, \ldots$, in $B_{k} \cup$ $\{b(S)\}$ into point sets $S_{1} \cup\{b(S)\}, \ldots, S_{k} \cup\{b(S)\}$, respectively.

If $s$ is not a cutvertex of $G$, consider the biconnected component $B$ incident to $s$.

If $B$ is an edge $\left(s, s^{\prime}\right)$, denote by $S^{\prime}$ the point set obtained by removing $b(S)$ from $S$ and by $G^{\prime}$ the graph obtained by removing $s$ and its incident edge from $G$ (see Fig.2 2 b)). Recursively construct an upward straight-line embedding of $G^{\prime}$ into $S^{\prime}$.

If $B$ is a 3-cycle $\left(s, s^{\prime}, s^{\prime \prime}\right)$, denote by $b^{*}(S)$ the lowest point of $S$ different from $b(S)$. Denote by $G^{\prime}$ (by $G^{\prime \prime}$ ) the graph composed of $s^{\prime}$ (resp. of $s^{\prime \prime}$ ) and of every connected component not containing $s$ that is obtained by removing $s^{\prime}$ (resp. $s^{\prime \prime}$ ) from $G$. Clockwise separate $\left|G^{\prime}\right|-1$ points around $b^{*}(S)$. Such points, together with $b^{*}(S)$, form a set $S^{\prime}$. Let $S^{\prime \prime}=S \backslash\left\{S^{\prime} \cup\{b(S)\}\right\}$. Consider a line $l$ fixed at $b^{*}(S)$ and rotating clockwise starting from a horizontal position. If $l$ encounters $b\left(S^{\prime \prime}\right)$ after $s$ (see Fig. 2(c)), then recursively construct upward straight-line embeddings of $G^{\prime}$ into $S^{\prime}$ and of $G^{\prime \prime}$ into $S^{\prime \prime}$. If $l$ encounters $b\left(S^{\prime \prime}\right)$ before $s$ (see Fig.2(d)), then counterclockwise separate $\left|G^{\prime}\right|-1$ points around $b^{*}(S)$. Such points, together with $b^{*}(S)$, form a set $S_{1}$. Let $S_{2}=S \backslash\left\{S_{1} \cup\{b(S)\}\right\}$. Then, recursively construct upward straight-line embeddings of $G^{\prime}$ into $S_{1}$ and of $G^{\prime \prime}$ into $S_{2}$.

We get the following:

Theorem 2. Every single-source upward planar directed graph whose every simple cycle has length three admits an upward straight-line embedding into every point set in general position. 


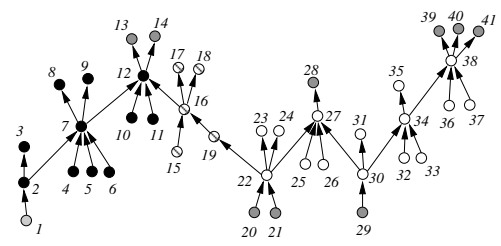

(a)

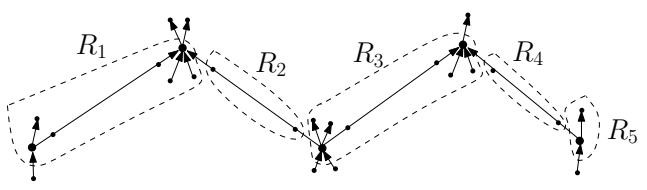

(b)

Fig. 3. (a) A caterpillar $G=(V, E)$ and function $\mathcal{E}: V \rightarrow\{1,2, \ldots, n\}$. (b) Decomposition of a caterpillar into increasing caterpillars, decreasing caterpillars, and extremal legs.

\section{Embeddings of Directed Caterpillars into Convex Point Sets}

In this section we prove that every caterpillar admits an upward straight-line embedding into every point set in convex position.

We introduce some terminology. A caterpillar $G$ is a tree such that removing all the degree-1 vertices, called the legs of $G$, yields a path, called the spine of $G$. A caterpillar whose spine is a monotone path is a monotone caterpillar. Let $v_{s}$ and $v_{t}$ be a source and a sink of the spine of a caterpillar. A vertex $w$ that is connected to $v_{s}$ by edge $\left(w, v_{s}\right)$ or to $v_{t}$ by edge $\left(v_{t}, w\right)$ is an extremal leg of a caterpillar. In Fig. 3. a) the extremal legs are numbered 1, 13, 14, 20, 21, 28, 29, 39, 40, 41.

Let $G$ be a caterpillar, let $T$ be its spine, and let $u$ be one of the end-points of $T$. Let $U$ be the set composed of $u$ and of the extremal legs of $G$ adjacent to $u$. The following lemma descends from algorithms presented in the literature [8]3].

Lemma 3. Suppose that $u$ is a source (resp. a sink) of T. Then, $G$ admits an upward straight-line embedding into every one-side convex point set $S$ in which the vertices of $G$ in $U$ are mapped to the $|U|$ lowest (resp. highest) points of $S$.

Let $G$ be a monotone caterpillar and let $T$ be its spine. Let $s$ and $t$ be the source and the sink of $T$, respectively. In addition, suppose that $s$ and $t$ are a source and a sink of $G$, respectively. We have the following:

Lemma 4. G admits an upward straight-line embedding into every convex point set $S$ in which $s$ is mapped to the lowest point of $S$ and $t$ is mapped to the highest point of $S$.

Next, for a caterpillar $G=(V, E)$, we define a bijective function $\mathcal{E}: V \rightarrow\{1,2, \ldots, n\}$. Let $T$ be the spine of $G$ and let $a$ and $b$ be the end-vertices of $T$. Function $\mathcal{E}$ is defined according to the following rules (see Fig. $3(\mathrm{a})$ ). ( $\mathcal{R} 1$ ): For any two vertices $u, v \in T$ such that $u$ comes before $v$ when traversing $T$ from $a$ to $b$, the value associated to $u$ and to all the legs adjacent to $u$ is smaller than the value associated to $v$ and to all legs adjacent to $v$; $(\mathcal{R} 2)$ : For any vertex $u \in T$, the value associated to $u$ is greater than the value associated to all the legs incident to edges entering $u$; $(\mathcal{R} 3)$ : For any vertex $u \in T$, the value associated to $u$ is smaller than the value associated to all the legs incident to edges exiting $u$.

We now describe an algorithm to construct an upward straight-line embedding of any caterpillar $G$ into any convex point set $S$. The idea is to partition $G$ into three 


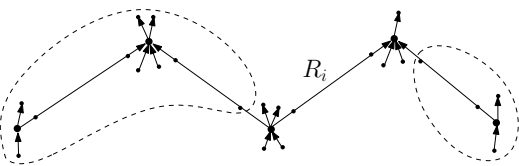

(a)

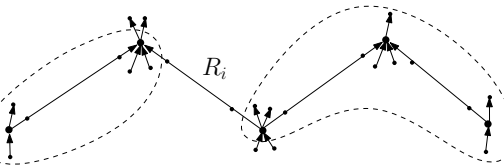

(b)

Fig. 4. Sub-caterpillars of (a) an increasing caterpillar $R_{i}$ and (b) a decreasing caterpillar $R_{i}$

smaller caterpillars that can be embedded into suitable subsets of $S$ by means of the two algorithms described above. In the following we formalize this idea.

Let $G$ be a caterpillar with spine $T$. Let $\left(T_{1}, \ldots, T_{k}\right)$ be the maximal monotone paths composing $T ; T_{i}$ is an increasing path if, for any edge $(u, v)$ in $T_{i}, \mathcal{E}(u)<\mathcal{E}(v)$ and a decreasing path otherwise. Assume the sources and the sinks of $T$ belong to the increasing paths and not to the decreasing paths. Hence, $T_{1}$ and $T_{k}$ are increasing paths, possibly with one vertex. Let $R_{i}$ be the caterpillar induced by $T_{i}$ and by the nonextremal legs of $G$ adjacent to $T_{i} ; R_{i}$ is an increasing (resp. decreasing) caterpillar if $T_{i}$ is an increasing (resp. decreasing) path. Caterpillar $G$ is partitioned into increasing caterpillars, decreasing caterpillars, and extremal legs (see Fig. 3 (b)). If $R_{i}$ is an increasing caterpillar, let $s\left(R_{i}\right)$ and $t\left(R_{i}\right)$ be the source and the sink of $T_{i}$, respectively. If $R_{i}$ is a decreasing caterpillar, let $t\left(R_{i}\right)=t\left(R_{i-1}\right)$ and $s\left(R_{i}\right)=s\left(R_{i+1}\right)$, that is, $s\left(R_{i}\right)$ (resp. $t\left(R_{i}\right)$ ) is the source (the sink) of $T$ immediately following (preceding) $T_{i}$. Observe that, if $R_{i}$ is a decreasing caterpillar, $s\left(R_{i}\right)$ and $t\left(R_{i}\right)$ do not belong to $R_{i}$.

Next, we define the sub-caterpillars $G_{i}^{1}$ and $G_{i}^{2}$ of $G$ induced by $R_{i}$. If $R_{i}$ is an increasing caterpillar (see Fig. 4(a)), set $G_{i}^{1}\left(G_{i}^{2}\right)$ to be the caterpillar induced by the vertices of $G$ preceding $s\left(R_{i}\right)$ (following $t\left(R_{i}\right)$, resp.) in $\mathcal{E}$, except for the extremal legs adjacent to $s\left(R_{i}\right)$ (to $t\left(R_{i}\right)$, resp.). If $R_{i}$ is a decreasing caterpillar (see Fig. 4 (b)), set $G_{i}^{1}\left(G_{i}^{2}\right)$ to be the caterpillar induced by $t\left(R_{i}\right)$ (by $s\left(R_{i}\right)$, resp.) and by the vertices of $G$ preceding $t\left(R_{i}\right)$ (following $s\left(R_{i}\right)$, resp.) in $\mathcal{E}$.

Consider a line $l$ through $b(S)$ and $t(S)$. Denote by $A$ and $B$ the point sets to the left and to the right of $l$, resp. (see Fig.5(a)). Points $b(S)$ and $t(S)$ belong to $A$. Consider any increasing caterpillar $R_{i}$. Denote by $i_{l}$ (resp. $i_{h}$ ) the number of extremal legs adjacent to $s\left(R_{i}\right)$ (resp. to $t\left(R_{i}\right)$ ) and by $L$ (resp. $H$ ) the set of the $i_{l}+1$ lowest (resp. of the $i_{h}+1$ highest) points of $S$. Let $A^{\prime}=A \backslash(L \cup H), B^{\prime}=B \backslash(L \cup H),|H \cap A|=$ $h_{a},|H \cap B|=h_{b},|L \cap A|=l_{a}$, and $|L \cap B|=l_{b}$ (see Fig. 5a)). We have the following:

Lemma 5. If $\left|G_{i}^{1}\right| \leq|A|-l_{a}$ and $\left|G_{i}^{2}\right| \leq|B|-h_{b}$, then there is an upward straight-line embedding of $G$ into $S$.

Proof: We distinguish three cases:

Case 1: $\left|G_{i}^{1}\right| \leq\left|A^{\prime}\right|$ and $\left|G_{i}^{2}\right| \leq\left|B^{\prime}\right|$. Refer to Fig. 6(a). Map $s\left(R_{i}\right)$ to $t(L)$ and the extremal legs adjacent to $s\left(R_{i}\right)$ to the other points of $L$. Map $t\left(R_{i}\right)$ to $b(H)$ and the extremal legs adjacent to $t\left(R_{i}\right)$ to the other points of $H$. Embed $G_{i}^{1}$ into the $\left|G_{i}^{1}\right|$ lowest points of $A^{\prime}$ and $G_{i}^{2}$ into the $\left|G_{i}^{2}\right|$ highest points of $B^{\prime}$. Such embeddings can be constructed by Lemma 3 , since $A^{\prime}$ and $B^{\prime}$ are one-side convex point sets. Embed 


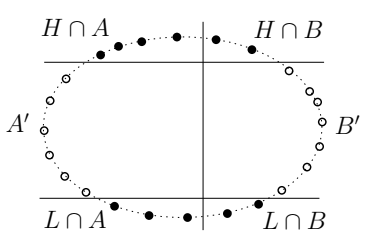

(a)

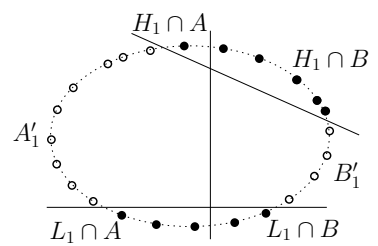

(b)

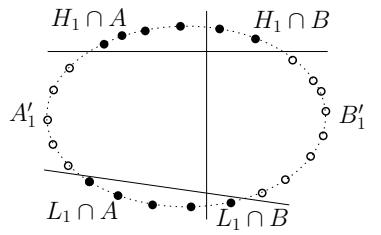

(c)

Fig. 5. Point sets for (a) Case 1, (b) Case 2, and (c) Case 3

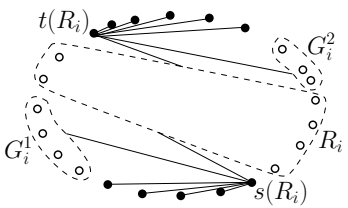

(a)

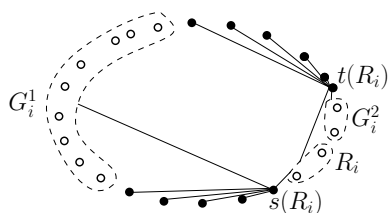

(b)

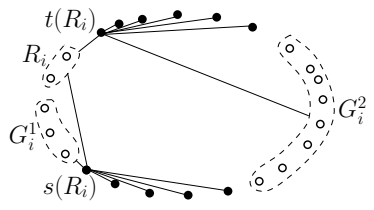

(c)

Fig. 6. Embedding $G$ into $S$ if (a) $\left|G_{i}^{1}\right| \leq\left|A^{\prime}\right|$ and $\left|G_{i}^{2}\right| \leq\left|B^{\prime}\right|$, (b) $\left|G_{i}^{1}\right| \leq|A|-l_{a},\left|G_{i}^{2}\right| \leq$ $|B|-h_{b}$, and $\left|G_{i}^{1}\right|>\left|A^{\prime}\right|$, and (c) $\left|G_{i}^{2}\right| \leq|B|-h_{b},\left|G_{i}^{1}\right| \leq\left|A^{\prime}\right|$, and $\left|G_{i}^{2}\right|>\left|B^{\prime}\right|$

$R_{i}$ into the remaining free points of $S$. This can be done by Lemma 4 since $R_{i}$ is a monotone caterpillar. We have the following:

Claim 1. The constructed straight-line embedding of $G$ into $S$ is upward and planar.

Case $2:\left|G_{i}^{1}\right|>\left|A^{\prime}\right|$. We create a partition $\left(A_{1}^{\prime}, B_{1}^{\prime}, H_{1}, L_{1}\right)$ of $S$ such that $\left|G_{i}^{1}\right|=\left|A_{1}^{\prime}\right|$, $\left|G_{i}^{2}\right|+\left|R_{i}\right|=\left|B_{1}^{\prime}\right|, i_{l}+1=\left|L_{1}\right|$, and $i_{h}+1=\left|H_{1}\right|$ (see Fig. 5(b)). Let $d_{G_{i}^{1}}=\left|G_{i}^{1}\right|-$ $\left|A^{\prime}\right|$. By the assumptions of the lemma, $\left|G_{i}^{1}\right| \leq|A|-l_{a}$. Since $|A|=\left|A^{\prime}\right|+l_{a}+h_{a}$, we have $d_{G_{i}^{1}} \leq h_{a}$. Define $A_{1}^{\prime}$ as $A^{\prime}$ plus the $d_{G_{i}^{1}}$ lowest points of $H \cap A, B_{1}^{\prime}$ as $B^{\prime}$ minus the $d_{G_{i}^{1}}$ highest points of $B^{\prime}, L_{1}=L$, and $H_{1}^{i}=S \backslash\left(A_{1}^{\prime} \cup B_{1}^{\prime} \cup L_{1}\right)$. Refer to Fig.6(b). Map $s\left(R_{i}\right)$ to $t\left(L_{1}\right)$ and map the extremal legs adjacent to $s\left(R_{i}\right)$ to the other points of $L_{1}$. Map $t\left(R_{i}\right)$ to $b\left(H_{1}\right)$ and the extremal legs adjacent to $t\left(R_{i}\right)$ to the other points of $H_{1}$. Embed $G_{i}^{1}$ into $A_{1}^{\prime}$ and $G_{i}^{2}$ into the $\left|G_{i}^{2}\right|$ highest points of $B_{1}^{\prime}$. Such embeddings can be constructed by Lemma 3 , since $A_{1}^{\prime}$ and $B_{1}^{\prime}$ are one-side convex point sets. Embed $R_{i}$ into the remaining free points of $S$. This can be done by Lemma 4 since $R_{i}$ is a monotone caterpillar. We have the following:

Claim 2. The constructed straight-line embedding of $G$ into $S$ is upward and planar.

Case 3: $\left|G_{i}^{1}\right| \leq\left|A^{\prime}\right|$ and $\left|G_{i}^{2}\right|>\left|B^{\prime}\right|$. We create a partition $\left(A_{1}^{\prime}, B_{1}^{\prime}, H_{1}, L_{1}\right)$ of $S$ such that $\left|G_{i}^{1}\right|+\left|R_{i}\right|=\left|A_{1}^{\prime}\right|,\left|G_{i}^{2}\right|=\left|B_{1}^{\prime}\right|, i_{l}+1=\left|L_{1}\right|$, and $i_{h}+1=\left|H_{1}\right|$ (see Fig. 5(c)). Let $d_{G_{i}^{2}}=\left|G_{i}^{2}\right|-\left|B^{\prime}\right|$. By the assumptions of the lemma, $\left|G_{i}^{2}\right| \leq|B|-h_{b}$. Since $|B|=\left|B^{\prime}\right|^{2}+l_{b}+h_{b}$, we have $d_{G_{i}^{2}} \leq l_{b}$. Define $B_{1}^{\prime}$ as $B^{\prime}$ plus the $d_{G_{i}^{2}}$ highest points of $L \cap B, A_{1}^{\prime}$ as $A^{\prime}$ minus the $d_{G_{i}^{2}}$ lowest points of $A^{\prime}, H_{1}=H$, and $L_{1}=$ $S \backslash\left(A_{1}^{\prime} \cup B_{1}^{\prime} \cup H_{1}\right)$. Refer to Fig. 6(c). Map $s\left(R_{i}\right)$ to $t\left(L_{1}\right)$ and the extremal legs 
adjacent to $s\left(R_{i}\right)$ to the other points of $L_{1}$. Map $t\left(R_{i}\right)$ to $b\left(H_{1}\right)$ and the extremal legs adjacent to $t\left(R_{i}\right)$ to the other points of $H_{1}$. Embed $G_{i}^{2}$ into $B_{1}^{\prime}$ and $G_{i}^{1}$ into the $\left|G_{i}^{1}\right|$ lowest points of $A_{1}^{\prime}$. Such embeddings can be constructed by Lemma 3 , since $A_{1}^{\prime}$ and $B_{1}^{\prime}$ are one-side convex point sets. Embed $R_{i}$ into the remaining free points of $S$. This can be done by Lemma 4 , since $R_{i}$ is a monotone caterpillar. We have the following:

Claim 3. The constructed straight-line embedding of $G$ into $S$ is upward and planar.

Now consider any decreasing caterpillar $R_{i}$ that is part of $G$. Define $i_{l}, i_{h}, L, H, A^{\prime}$, $B^{\prime}, h_{a}, h_{b}, l_{a}$, and $l_{b}$ as before. We have the following:

Lemma 6. If $\left|G_{i}^{1}\right| \leq|A|-h_{a}+1$ and $\left|G_{i}^{2}\right| \leq|B|-l_{b}+1$, then there is an upward straight-line embedding of $G$ into $S$.

The proof of Lemma 6 is analogous to the one of Lemma 5 The requirements $\left|G_{i}^{1}\right| \leq$ $|A|-h_{a}+1$ and $\left|G_{i}^{2}\right| \leq|B|-l_{b}+1$ of Lemma 6 are weaker than the requirements $\left|G_{i}^{1}\right| \leq|A|-l_{a}$ and $\left|G_{i}^{2}\right| \leq|B|-h_{b}$ of Lemma 5 This is due to the fact that, if $R_{i}$ is a decreasing caterpillar, then $t\left(R_{i}\right)$ and $s\left(R_{i}\right)$ belong to $G_{i}^{1}$ and to $G_{i}^{2}$, respectively, and they do not belong to $R_{i}$. We are now ready to prove the following:

Theorem 3. Any n-vertex directed caterpillar $G$ admits an upward straight-line embedding into every convex point set $S$ with $n$ points.

Proof: Let $T$ be the spine of $G$ and let $\left\{R_{1}, \ldots, R_{k}\right\}$ be the increasing and decreasing caterpillars of $G$. Let $(A, B)$ be the partition of $S$ created by a line through $b(S)$ and $t(S)$, where $b(S)$ and $t(S)$ belong to $A$. Consider the $|A|$-th vertex $v_{|A|}$ in the order $v_{1}, \ldots, v_{n}$ of the vertices of $G$ defined by $\mathcal{E}$. We partition $G$ into three smaller caterpillars and we draw each of them on a suitably chosen portion of $S$. The partition of $G$ is determined by the position of $v_{|A|}$ in $G$. We distinguish four cases:

Case 1: $v_{|A|}$ is a vertex of an increasing caterpillar $R_{i}$. Define $i_{l}, i_{h}, L, H, h_{b}, l_{a}, h_{a}$, and $l_{b}$ as before. Since $v_{|A|} \in R_{i}$, we have that $\left|G_{i}^{1}\right|+i_{l}<|A|$. Since $i_{l}+1=l_{a}+l_{b}$, it follows that $l_{a} \leq i_{l}+1$. Thus, $\left|G_{i}^{1}\right|+l_{a} \leq|A|$. Analogously, we have that $\left|G_{i}^{2}\right|+i_{h}<$ $|B|$. Since $i_{h}+1=h_{a}+h_{b}$, it follows that $h_{b} \leq i_{h}+1$. Thus, $\left|G_{i}^{2}\right|+h_{b} \leq|B|$. Hence, Lemma 5 applies and the result follows.

Case 2: $v_{|A|}$ is a vertex of a decreasing caterpillar $R_{i}$. Analogously to Case 1 , it can be proven that $\left|G_{i}^{1}\right| \leq|A|-h_{a}+1$ and $\left|G_{i}^{2}\right| \leq|B|-l_{b}+1$. Hence, Lemma 6 applies and the result follows.

Case 3: $v_{|A|}$ is an extremal leg adjacent to a sink of $T$. Let $R_{i}$ and $R_{i+1}$ be such that $t\left(R_{i}\right)=t\left(R_{i+1}\right)$ and $v_{|A|}$ is an extremal leg adjacent to $t\left(R_{i}\right)$. Note that $R_{i}$ is an increasing caterpillar and $R_{i+1}$ is a decreasing caterpillar. Denote by $i_{h}$ the number of extremal legs adjacent to $t\left(R_{i}\right)$ and by $H$ the set of the $i_{h}+1$ highest points of $S$. Let $|H \cap B|=h_{b}$ and $|H \cap A|=h_{a}$. Notice that $h_{a}+h_{b}=i_{h}+1$. We claim the following.

Claim 4. Let $G_{i}^{1}$ and $G_{i}^{2}$ (let $G_{i+1}^{1}$ and $G_{i+1}^{2}$ ) be the sub-caterpillars of $G$ induced by $R_{i}$ (resp. by $R_{i+1}$ ). At least one of the following inequalities holds: (1) $\left|G_{i+1}^{1}\right| \leq$ $|A|-h_{a}+1 ;(2)\left|G_{i}^{2}\right| \leq|B|-h_{b}$. 
We further distinguish two cases.

Inequality (1) holds. Consider the decreasing caterpillar $R_{i+1}$. Let $i_{l}$ be the number of extremal legs adjacent to $s\left(R_{i+1}\right)$ and let $L$ be the set of the $i_{l}+1$ lowest point of $S$. Denote $l_{a}=|L \cap A|$ and $l_{b}=|L \cap B|$. We have that $|B|>\left|G_{i+1}^{1}\right|+i_{l}$, hence $|B| \geq\left|G_{i+1}^{1}\right|+l_{b}$, as $i_{l}+1=l_{a}+l_{b}$. Hence, Lemma 6 applies and the result follows.

Inequality (2) holds. Consider the increasing caterpillar $R_{i}$. Let $i_{l}$ be the number of extremal legs adjacent to $s\left(R_{i+1}\right)$ and let $L$ be the set of the $i_{l}+1$ lowest point of $S$. Denote $l_{a}=|L \cap A|$ and $l_{b}=|L \cap B|$. We have that $|A|>\left|G_{i}^{1}\right|+i_{l}$, hence $|A| \geq\left|G_{i}^{1}\right|+l_{a}$, as $i_{l}+1=l_{a}+l_{b}$. Hence, Lemma 5 applies and the result follows.

Case 4: $v_{|A|}$ is an extremal leg adjacent to a source of $T$. Analogously to Case 3, it can be proven that either $|A| \geq\left|G_{i+1}^{1}\right|+h_{a}-1$ and $|B| \geq\left|G_{i+1}^{1}\right|+l_{b}-1$ hold simultaneously, thus the result follows from Lemma 6 or $|A| \geq\left|G_{i}^{1}\right|+l_{a}$ and $|B| \geq$ $\left|G_{i}^{2}\right|+h_{b}$ hold simultaneously, thus the result follows from Lemma 5

\section{Embeddings of Directed Paths into General Point Sets}

In this section we deal with upward straight-line embeddings of paths with few switches into general point sets. We first deal with paths with four switches.

Theorem 4. Every path $P$ composed of three monotone paths $P_{1}, P_{2}$, and $P_{3}$ admits an upward straight-line embedding into every general point set $S$ if at least one out of $P_{1}, P_{2}$, and $P_{3}$ is a trivial path.

Proof: Let $P_{1}=\left(s_{1}=u_{1}, \ldots, u_{U}=t_{1}\right), P_{2}=\left(t_{1}=v_{1}, \ldots, v_{V}=s_{2}\right)$, and $P_{3}=\left(s_{2}=w_{1}, \ldots, w_{W}=t_{2}\right)$ be the monotone paths composing $P$, where $s_{1}$ and $s_{2}$ are sources and $t_{1}$ and $t_{2}$ are sinks. If $P_{2}$ is trivial, a more general result in [3] states that a path $P$ admits an upward straight-line embedding into every general point set if the $i$-th monotone path composing $P$ is trivial, for every odd $i$ or for every even $i$. We discuss the case in which $P_{3}$ is trivial, the case in which $P_{1}$ is trivial being symmetric. Counterclockwise separate a set $S_{1}$ of $U-1$ points around $t(S)$. Construct upward straight-line embeddings of $P_{1}$ into $S_{1} \cup\{t(S)\}$ and of $P_{2} \backslash\left\{s_{2}\right\}$ into the $V-1$ highest points of $S \backslash S_{1}$. Map $s_{2}$ to $b\left(S \backslash S_{1}\right)$ and $t_{2}$ to the only remaining free point of $S$.

Claim 5. The constructed straight-line embedding of $P$ into $S$ is upward and planar.

We now deal with paths with five switches.

Theorem 5. Every path $P$ composed offour monotone paths $P_{1}, P_{2}, P_{3}$, and $P_{4}$ admits an upward straight-line embedding into every general point set $S$ if at least two out of $P_{1}, P_{2}, P_{3}$, and $P_{4}$ are trivial paths.

Proof: Let $P_{1}=\left(s_{1}=u_{1}, u_{2}, \ldots, u_{U}=t_{1}\right), P_{2}=\left(t_{1}=v_{1}, v_{2}, \ldots, v_{V}=s_{2}\right)$, $P_{3}=\left(s_{2}=w_{1}, w_{2}, \ldots, w_{W}=t_{2}\right)$, and $P_{4}=\left(t_{2}=z_{1}, z_{2}, \ldots, z_{Z}=s_{3}\right)$ be the monotone paths composing $P$, where $s_{1}, s_{2}$, and $s_{3}$ are sources and $t_{1}$ and $t_{2}$ are sinks. The case in which $P$ has three sinks and two sources can be discussed analogously. If $P_{1}$ and $P_{3}$ are trivial or if $P_{2}$ and $P_{4}$ are trivial, the proof follows from the result in [3] 
cited in the proof of Theorem 4 We discuss the case in which $P_{1}$ and $P_{4}$ are trivial. Clockwise separate a set $S_{1,2}$ of $V$ points around $b(S)$. Construct upward straight-line embeddings of $P_{2} \backslash\left\{t_{1}\right\}$ into the $V-1$ lowest points of $S_{1,2} \cup b(S)$ and of $P_{3} \backslash\left\{t_{2}\right\}$ into the $W-1$ lowest points of $S \backslash S_{1,2}$. Map $t_{1}$ to $t\left(S_{1,2}\right)$ and $s_{1}$ to the only remaining free point of $S_{1,2}$. Map $t_{2}$ to $t\left(S \backslash S_{1,2}\right)$ and $s_{3}$ to the only remaining free point of $S \backslash S_{1,2}$.

\section{Claim 6. The constructed straight-line embedding of $P$ into $S$ is upward and planar.}

We discuss the case in which $P_{1}$ and $P_{2}$ are trivial, the case in which $P_{3}$ and $P_{4}$ are trivial being symmetric. Clockwise separate a set $S_{4}$ of $Z-1$ points around $t(S)$. Construct upward straight-line embeddings of $P_{4}$ into $S_{4} \cup\{t(S)\}$ and of $P_{3} \backslash\left\{s_{2}\right\}$ into the $W-1$ highest points of $S \backslash S_{4}$. Consider the line $l^{\prime}$ through the point where $w_{2}$ is drawn and the lowest free point of $S$. If both the two remaining free points of $S$ are on the same side of $l^{\prime}$, then map $s_{2}$ to $b\left(S \backslash S_{4}\right)$, map $t_{1}$ to the highest free point of $S$, and map $s_{1}$ to the other free point of $S$. Otherwise, one of the two remaining free points of $S$ is to the left $l^{\prime}$ and the other one is to its right. Then, map $s_{2}$ to the lowest of such two points, map $t_{1}$ to the highest of such two points, and map $s_{1}$ to $b\left(S \backslash S_{4}\right)$.

Claim 7. The constructed straight-line embedding of $P$ into $S$ is upward and planar.

We discuss the more involved case in which $P_{2}$ and $P_{3}$ are trivial. Let $p_{1}$ and $p_{2}$ be the two highest points of $S$, with $y\left(p_{1}\right) \geq y\left(p_{2}\right)$. Counterclockwise separate a set $S_{1}$ of $U-1$ points around $p_{2}$. Denote by $p$ the point that is added to $S_{1}$ when $U$ points are counterclockwise separated around $p_{2}$. We consider the following two cases:

Point $p$ is to the left of $l_{1,2}$ : Refer to Fig. 7 (7a). Consider a half-line $l_{1}$ fixed at $p_{1}$ and passing through $p$. Rotate $l_{1}$ in counterclockwise direction. Let $p^{\prime}$ be the last point of $S_{1}$ encountered by $l_{1}$ before encountering $p_{2}$. If no point of $S_{1}$ is encountered by $l_{1}$ before $p_{2}$, then let $p^{\prime}=p$. Map $t_{1}$ to $p_{1}, t_{2}$ to $p_{2}$, and $s_{2}$ to $p^{\prime}$; construct upward straight-line embeddings of $P_{1}$ into $S_{1} \cup\left\{p_{1}, p\right\} \backslash\left\{p^{\prime}\right\}$ and of $P_{4}$ into $S \backslash\left\{S_{1}\right\} \cup\left\{p_{2}\right\} \backslash\{p\}$.

Point $p$ is to the right of $l_{1,2}$ : Refer to Fig. 7(b). Consider a half-line $l_{1}$ fixed at $p_{1}$ and passing through $p$. Rotate $l_{1}$ in clockwise direction. Let $p^{\prime}$ be the last point of $S_{1}$ encountered by $l_{1}$ before encountering $p_{2}$. If no point of $S_{1}$ is encountered by $l_{1}$ before $p_{2}$, then let $p^{\prime}=p$. Map $t_{1}$ to $p_{2}, t_{2}$ to $p_{1}$, and $s_{2}$ to $p^{\prime}$; construct upward straight-line embeddings of $P_{1}$ into $S_{1} \cup\left\{p_{2}\right\}$ and of $P_{4}$ into $S \backslash\left\{S_{1}\right\} \cup\left\{p_{1}, p\right\} \backslash\left\{p^{\prime}\right\}$.

Claim 8. The constructed straight-line embedding of $P$ into $S$ is upward and planar.

Next, we tackle the problem of embedding paths with at most $k$ switches into general point sets with more than $n$ points. We show the following:

Theorem 6. Every directed path $P$ with $n$ vertices and $k$ switches admits an upward straight-line embedding into every general point set $S$ with $|S| \geq n 2^{k-2}$.

Proof: We prove the statement by induction on the number of switches; we suppose inductively that one of the end-vertices of $P$ is mapped to $b(S)$ or $t(S)$, depending on whether such a vertex is a source or a sink. The statement is trivial if $k=2$, as in such a case $P$ is monotone and any general point set with $n$ points suffices. 


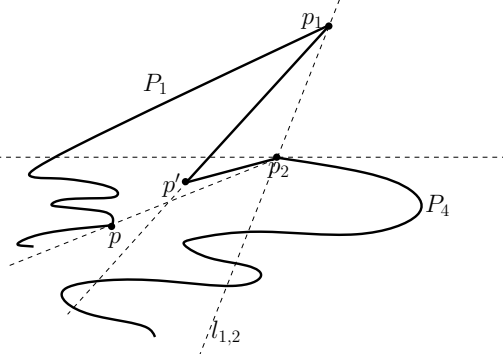

(a)

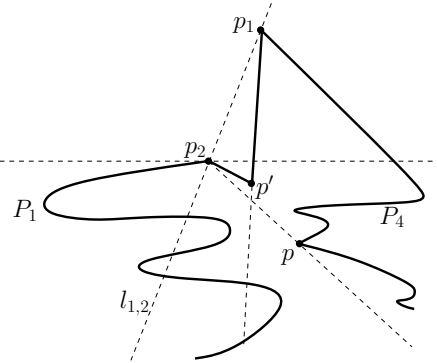

(b)

Fig. 7. (a) Point $p$ is to the left of $l_{1,2}$. (b) Point $p$ is to the right of $l_{1,2}$.

Suppose that $k>2$. Let $a_{1}$ be an end-vertex of $P$. Suppose that $a_{1}$ is a source, the case in which it is a sink being analogous. Let $P_{a}=\left(a_{1}, a_{2}, \ldots, a_{l}\right)$ be the maximal monotone path of $P$ containing $a_{1}$. Notice that $l \geq 2$. Map $P_{a} \backslash\left\{a_{l}\right\}$ to the $\left|P_{a}\right|-1$ points of $S$ with lowest $y$-coordinate. Denote such a point set by $S_{a}$. Map $a_{l}$ to $t(S)$. Let $S_{1}$ and $S_{2}$ be the point sets composed of $t(S)$ and of the points of $S \backslash S_{a}$ to the left and to the right, respectively, of the line through $a_{l-1}$ and $a_{l}$. If $\left|S_{1}\right| \geq\left|S_{2}\right|$ (if $\left|S_{2}\right|>\left|S_{1}\right|$ ), construct an upward straight-line embedding of $P \backslash\left\{a_{1}, a_{2}, \ldots, a_{l-1}\right\}$ into $S_{1}$ (into $S_{2}$, resp.) with $a_{l}$ placed at $t\left(S_{1}\right)=t(S)$ (at $t\left(S_{2}\right)=t(S)$, resp.).

It is easy to see that the constructed straight-line embedding is upward and planar. We show that the cardinality of point sets $S_{1}$ and $S_{2}$ is sufficient to apply the induction. The number of points in the one of $S_{1}$ and $S_{2}$ with more points is at least $(|S|-(l-1)) / 2$. Further, $P \backslash\left\{a_{1}, a_{2}, \ldots, a_{l-1}\right\}$ has $n-(l-1)$ vertices and $k-1$ switches. Since $|S| \geq n 2^{k-2}$, the one of $S_{1}$ and $S_{2}$ with more points has at least $\left(n 2^{k-2}-(l-1)\right) / 2=$ $n 2^{k-3}-(l-1) / 2>n 2^{k-3}-(l-1) 2^{k-3}$ and, since $k>2$, the lemma follows.

\section{Open Problems}

In this paper we continued the study of upward straight-line embeddability of directed graphs into point sets initiated in [8]3]. While we solved some of the open questions posed by Binucci et al. in [3], the following problems remain open: (i) Is it possible to test in polynomial time whether a directed graph/tree admits an upward straight-line embedding into every point set in general/convex position? (ii) Does every directed path admit an upward straight-line embedding into every point set in general position? (iii) Is there a polynomial function $p(n, k)$ such that every directed path admits an upward straight-line embedding into every point set in general position with at least $p(n, k)$ points? Lemma 6 shows that every directed path admits an upward straight-line embedding into every point set in general position with at least $n 2^{k-2}$ points, which is exponential in $k$.

\section{References}

1. Angelini, P., Frati, F., Geyer, M., Kaufmann, M., Mchedlidze, T., Symvonis, A.: Upward geometric graph embeddings into point sets. Tech. Report 177, Dipartimento di Informatica e Automazione, Università Roma Tre (2010) 
2. Badent, M., Di Giacomo, E., Liotta, G.: Drawing colored graphs on colored points. Theor. Comput. Sci. 408(2-3), 129-142 (2008)

3. Binucci, C., Di Giacomo, E., Didimo, W., Estrella-Balderrama, A., Frati, F., Kobourov, S., Liotta, G.: Upward straight-line embeddings of directed graphs into point sets. Computat. Geom. Th. Appl. 43, 219-232 (2010)

4. Bose, P.: On embedding an outer-planar graph in a point set. Computat. Geom. Th. Appl. 23(3), 303-312 (2002)

5. Bose, P., McAllister, M., Snoeyink, J.: Optimal algorithms to embed trees in a point set. J. Graph Alg. Appl. 1(2), 1-15 (1997)

6. Cabello, S.: Planar embeddability of the vertices of a graph using a fixed point set is NP-hard. J. Graph Alg. Appl. 10(2), 353-366 (2006)

7. Di Giacomo, E., Didimo, W., Liotta, G., Meijer, H., Trotta, F., Wismath, S.K.: k-colored point-set embeddability of outerplanar graphs. J. Graph Alg. Appl. 12(1), 29-49 (2008)

8. Giordano, F., Liotta, G., Mchedlidze, T., Symvonis, A.: Computing upward topological book embeddings of upward planar digraphs. In: Tokuyama, T. (ed.) ISAAC 2007. LNCS, vol. 4835, pp. 172-183. Springer, Heidelberg (2007)

9. Gritzmann, P., Pach, B.M.J., Pollack, R.: Embedding a planar triangulation with vertices at specified positions. Amer. Math. Mont. 98, 165-166 (1991)

10. Kaufmann, M., Wiese, R.: Embedding vertices at points: Few bends suffice for planar graphs. J. Graph Alg. Appl. 6(1), 115-129 (2002)

11. Wiegers, M.: Recognizing outerplanar graphs in linear time. In: Tinhofer, G., Schmidt, G. (eds.) WG 1986. LNCS, vol. 246, pp. 165-176. Springer, Heidelberg (1987) 